\title{
Spaces of the Haar Type on Arbitrary Irregular Grids
}

\author{
YU.K.DEM'YANOVICH, I.G.BUROVA \\ Dept. of Parallel Computations, Dept. of Computational Mathematics \\ St. Petersburg State University \\ St. Petersburg, 7/9 Universitetskaya nab., \\ St.Petersburg, 199034 \\ RUSSIA
}

\begin{abstract}
The paper deals with Haar-type spaces on arbitrary irregular grids. The choice of non-uniform grids determines the characteristics of the Haar-type space that can be used to construct the wavelet decomposition. Thus, it becomes a possible adaptive choice of the design space depending on the incoming flow. In contrast to the classical approach, this paper considers the possibility of the adaptive compression of the initial flow. The complexity of the algorithm is directly proportional to the length of the initial number flow. Numerical examples are presented.
\end{abstract}

Key-Words: - Haar type spaces, pseudo-measure, adaptive grids, the computational complexity of the approximation

Received: April 9, 2020. Revised: September 23, 2020. Accepted: October 17, 2020. Published: November 5, 2020.

\section{Introduction}

Haar wavelets are often used in the numerical solution of differential and integro-differential equations. The solutions of partial differential equations which are not smooth enough, when approximated by cubic, quadratic and linear polynomials, results in poor convergence or no convergence in the results. In such cases, the approximation with Haar-type wavelets (piecewise continuous functions with finite jumps), are more suitable and successful. Numerical methods based on the Haar wavelet for solving the third-order Harry Dym (HD), Benjamin-Bona-MahonyBurger's (BBM Burger's) equation and 2D diffusion equation are developed in paper [1].

In study [2], the Haar wavelet collocation method is used for the numerical solution of $1 \mathrm{D}$ and 2D cubic nonlinear Schrodinger equations with initial and Dirichlet boundary conditions. An efficient collocation method for the numerical solution of the second and fourth order two-point boundary value problems (B.V.P.) based on the uniform Haar wavelet is proposed in [3]. Haar wavelets are used in signal and image processing. In paper [4] the Haar wavelet collocation technique is developed for the solution of nonlinear delay integro-differential equations for wireless sensor network and the industrial Internet of Things. The method is applied to the nonlinear delay Volterra, delay Fredholm and delay Volterra-Fredholm integro-differential equations which are based on the use of Haar wavelets. There are different techniques to approximate a signal using piecewise constant functions. The wavelet decomposition is one of them by means of a Haar wavelet. In paper [5], an optimal method of approximating a signal by piecewise constant functions is provided.

In the classical approach to the wavelet decomposition (see [7] - [8]) a standard thinning of the initial flow is usually performed (usually by removing the odd-numbered components), so getting a qualitative approximation of the initial flow is difficult. Within the framework of the classical approach in the work [8], wavelets are discussed on a nonuniform grid for the Haar functions (see [6]). In the mentioned work, the authors considered a non-uniform initial grid with the number of components $M=2^{s}$. Enlargement turned out removing the odd-numbered components. A number of nodes on the enlarged grid turned out to be equal to $K=2^{s-1}$. With the mentioned approach, the coarsening of the grid is not related to the properties of the initial flow, so hopefully the main flow is not a qualitative approximation of the initial one.

But a new type of the non-linear adaptive phaselocked loop (PLL), which is based on the spline interpolation has been proposed in [10]. The study 
discusses the method of the cubic spline, which is used on an irregular grid. In paper [11], a real-time technique to visualize large-scale adaptive height fields with the $C^{1}$-continuous surface reconstruction is presented. An adaptive phasor measurement units (PMU) missing data recovery method is proposed in paper [12]. In the paper an improved cubic spline interpolation based on the priority allocation strategy is proposed for ambient data loss, which can quickly and accurately recover ambient data. In [13] the formula for constructing the interpolation nodes for a rapidly increasing function or decreasing function is offered. The formula takes into account the local behaviour of the function on the previous three grid nodes, and it is based on the interpolation by the local quadratic polynomial splines. So, in [8] - [16], there is a tendency to improve the approximation of the data flow by choosing a nonuniform grid. The purpose of this work is to consider spaces of the Haar type on arbitrary irregular grids. With a non-classic wavelet approach [9], the choice of a non-uniform grid determines the characteristics space to be designed with wavelet decomposition. Thus, it becomes a possible adaptive choice of design space depending on the incoming flow. To expand the criteria adaptability in this work, the concept of a pseudo-measure is introduced. Two types of pseudo-measures are considered: a pseudo-measure, defined on the segments of the real axis, and a pseudo-measure, given on (so-called) grid segments. The relationships between the mentioned types of pseudo-measures are considered. Some concepts for the construction of the embedded Haar spaces with adaptivity properties in the norms of the spaces $\mathrm{C}$ are introduced.

The approximate properties of the constructed spaces are determined. The computational complexity of the obtained algorithms is proportional to the length of the incoming flow. This opens the way for the application of the nonclassical theory wavelets (see [16]).

\section{The Haar splines}

On the interval $(\alpha, \beta)$ of the real axis $R^{1}$ consider the grid

$$
\Xi: \ldots \xi_{-2}<\xi_{-1}<\xi_{0}<\xi_{1}<\xi_{2} \ldots
$$

with properties

$$
\lim _{i \rightarrow-\infty} \xi_{i}=\alpha, \lim _{i \rightarrow+\infty} \xi_{i}=\beta,
$$

We introduce piecewise constant functions $\omega_{j}(t)$, $t \in(\alpha, \beta)$, defined by equalities

$$
\omega_{j}(t)=\left\{\begin{array}{c}
1 \text { for } t \in\left[\xi_{j}, \xi_{j+1}\right) \\
0 \text { for } t \in R^{1} \backslash\left[\xi_{j}, \xi_{j+1}\right) .
\end{array}\right.
$$

System $\left\{\omega_{j}(t)\right\}_{j \in \mathbb{Z}}$ of functions (3) is defined by the grid (1)-(2) and is a linearly independent system.

The functions $\omega_{j}(t)$ are called coordinate splines of the zero order.

Remark. In the case when the grid $\Xi$ is uniform (and therefore $=-\infty, \beta=+\infty)$, the $\omega_{j}$ functions defined above are often called Haar functions.

Let $S_{j}=\left[\xi_{j}, \xi_{j+1}\right]$. It is clear that supp $\omega_{j}=S_{j}$. Approximation relation

$$
\sum_{j \in \mathbb{Z}} \omega_{j}(t)=1 \forall t \in(\alpha, \beta)
$$

in this case is the decomposition of unity into interval $(\alpha, \beta)$. For fixed $t \in(\alpha, \beta)$ the linear combination

$$
u(t)=\sum_{j \in \mathbb{Z}} c_{j} \omega_{j}(t)
$$

consists of one term: for $t \in\left[\xi_{j}, \xi_{j+1}\right.$ ) formula (4) takes the form $u(t)=c_{j} \omega_{j}(t)$.

Let $S_{0}(\Xi)$, defined by the relation

$S_{0}(\Xi)=C l_{p}\left\{u(t) \mid u(t)=\sum_{j} c_{j} \omega_{j}(t) \forall c_{j} \in R^{1}\right\}(5)$

is called the generalized Haar space on the grid $\Xi$, and the elements of this space are splines of the zero order. Symbol $\mathrm{Cl}_{p}$ in (5) denotes closure in the topology of pointwise convergence. Specifying the grid $\Xi$ uniquely determines the space $S_{0}(\Xi)$. The next point is about building embedded space splines of the zero order on a embedded grid.

\section{About Building an Embedded Grid}

If $\mathrm{c} \in \Xi$, then $i \in \mathbb{Z}$ exists such that $c=\xi_{i}$; in this case we denote $c^{-}=\xi_{i-1}, c^{+}=\xi_{i+1}$.

Let be $c, d \in \Xi, c^{+}<d^{-}$, i.e. for some $i, j \in \mathbb{Z}$, $i+2<j$, the equalities $\mathrm{c}=\xi_{i}, \mathrm{~d}=\xi_{j}$ are true. We will use the notation $|[c, d]|=\left\{\xi_{s} \mid c \leq \xi_{s} \leq\right.$ $d, s \in \mathbb{Z}\}$. A set of $|[c, d]|$ will be called a grid segment, and the set $\mid[c, d \mid[$ will be called a grid interval. It's clear that $\mid\left[c, d \mid\left[=\left|\left[c, d^{-}\right]\right|\right.\right.$.

The functions defined on the grid $\Xi$ will be called discrete functions (on a uniform grid, they are often called grid functions). The set of discrete functions $u(t)$ defined on the grid $\Xi$ is denoted $C(\Xi)$; it is clear that $C(E)$ is a linear space.

Also consider the linear normed space $C|[c, d]|$ of functions $u(t)$, defined on $|[c, d]|$. The norm is introduced by the ratio $\|u\|_{C|[c, d]|}=\max _{t \in[[c, d] \mid}|u(t)|$. 
In a similar way, we introduce the normed linear space $C \mid[c, d \mid[$ with the norm

$$
\|u\|_{C \mid[c, d \mid[}=\max _{t \in \mid[c, d \mid[}|u(t)| .
$$

Obviously, the spaces $C|[c, d]|$ and $C \mid[c, d \mid[$ are finite dimensional.

Let's denote $J_{m}=\{0,1,2, \ldots m\}$. Let $M$ be a natural number $M>2$ and $|[a, b]|=\left\{\xi_{s}\right\}_{s \in J_{M+1}}$, so that

$$
|[a, b]|=\left\{a=\xi_{0}<\cdots<\xi_{M}<\xi_{M+1}=b\right\} .
$$

A value $\tau=\max _{j \in J_{M}}\left(\xi_{j+1}-\xi_{j}\right)$ is called the maximum step in the grid segment $|[a, b]|$. Many grid segments, contained in the grid segment $|[a, b]|$ denote $\mathcal{O}$, so that

$$
\mathcal{O}=\mathcal{O}(|[a, b]|)=\{|[c, d]|||[c, d]|\subset|[a, b] \mid\} .
$$

Let $\mathcal{M}$ be some grid segment from the set $\mathcal{O}$.

Consider the mapping

$$
\begin{aligned}
& f: \mathcal{M} \rightarrow \mathbb{R}^{1}, \text { having the following } \\
& \qquad f(\mathcal{M}) \geq 0 \forall \mathcal{M} \subset \mathcal{O}, \\
& f(|[c, c]|)=0 \forall c \in|[a, b]|, \\
& \mathcal{M} \subset \mathcal{M} \mathcal{M}^{\prime} \Rightarrow f(\mathcal{M}) \leq f\left(\mathcal{M}^{\prime}\right) \forall \mathcal{M}, \mathcal{M}^{\prime} \subset \mathcal{O} .
\end{aligned}
$$

The mapping $f$ with the indicated properties will be called pseudo-measure on a grid segment $|[a, b]|$. Let a number $\varepsilon$ be in the interval $\left(\varepsilon^{*}, \varepsilon^{* *}\right)$,

$$
\varepsilon \in\left(\varepsilon^{*}, \varepsilon^{* *}\right) \text {, }
$$

where

$$
\varepsilon^{*}=\max _{\xi \in\left|\left[a, b^{-}\right]\right|} f\left(\left\{\xi, \xi^{+}\right\}\right), \varepsilon^{* *}=f(|[a, b]|) .
$$

Consider the grid $X \subset|[a, b]|$,

$$
\begin{aligned}
& X=X(f, \varepsilon,|[a, b]|): \\
& \quad a=x_{0}<x_{1}<\cdots<x_{K}<x_{K+1}=b
\end{aligned}
$$

such that

$$
\begin{aligned}
& f\left(\left|\left[x_{s}, x_{s+1}\right]\right|\right) \leq \varepsilon<f\left(\left|\left[x_{s}, x_{s+1}^{+}\right]\right|\right) \\
& \forall s \in\{0,1, \ldots K-1\}, \\
& f\left(\left|\left[x_{K}, b\right]\right|\right) \leq \varepsilon, X \subset|[a, b]| .
\end{aligned}
$$

Definition 1. Grid (11) with properties (12)-(13) is called an adaptive grid (for pseudo-measure $f$ on grid segment $|[a, b]|)$.

The following statement is true.

Theorem 1. If the relations (7)-(10) are satisfied, then a natural number $K=K(f, \varepsilon,|[a, b]|)$ : exists and a grid (11) with properties (12)-(13).

Proof. The proof is by induction.

I. The induction base is established as follows. We suppose that variable $\eta \in|[a, b]|$ increases from $a=x_{0}$ to $b$; then by assumptions (7)-(8) the function $\phi_{0}(\eta)=f\left(\left|\left[x_{0}, \eta\right]\right|\right)$ increases from 0 to $f(|[a, b]|)$. Due to the conditions (9)-(10) there is only one point $\eta_{1} \in|[a, b]|$ such that

$$
f\left(\left|\left[x_{0}, \eta_{1}\right]\right|\right) \leq \varepsilon<f\left(\left|\left[x_{0}, \eta_{1}^{+}\right]\right|\right) .
$$

Put $x_{1}=\eta_{1}$. The induction base is established.

II. Suppose the nodes $x_{1}, x_{2}, \ldots, x_{s}$ of the grid $X$ are defined. If $x_{s}=b$, then we put $K=s-1$. In this case, the building of the grid $X(f, \varepsilon,|[a, b]|)$ is completed. Otherwise $x_{s}<b$, and the grid continues. Consider the function $\phi_{s}(\eta)=$ $f\left(\left|\left[x_{s}, \eta\right]\right|\right)$. When $\eta$ changes from $x_{s}$ to $b$ function $\phi_{s}(\eta)$ grows from 0 to $m_{s}=f\left(\left|\left[x_{s}, b\right]\right|\right)$. Note that if $x_{s}=b^{-}$, then $m_{s}=f\left(\left\{b^{-}, b\right\}\right) \leq$ $\max _{\xi \in\left[\left[a, b^{-}\right] \mid\right.} f\left(\left\{\xi, \xi^{+}\right\}\right)=\varepsilon^{*}$,

and by assumptions (9)-(10) we have $m_{s} \leq \varepsilon$. In all cases when $m_{s} \leq \varepsilon$, we set $K=s$ and $x_{s+1}=b$.

Consider the case when $\varepsilon<m_{s}$; from the previous discussion it follows, that $x_{s}<b^{-}$. Since $0<\varepsilon<m_{s}$ and the discrete function $\phi_{s}(\eta)$ takes increasing values from 0 to $m_{s}$, then there is a single integer $j$ such that $\xi_{j} \in\left|\left[x_{s}, b^{-}\right]\right|$and $\phi_{s}\left(\xi_{j}\right) \leq \varepsilon<$ $\phi_{s}\left(\xi_{j+1}\right)$. This is a double inequality equivalent to the ratio $f\left(\left|\left[x_{s}, \xi_{j}\right]\right|\right) \leq \varepsilon<f\left(\left|\left[x_{s}, \xi_{j}^{+}\right]\right|\right)$.

Put $x_{s+1}=\xi_{j}$. Existence and uniqueness of the point $x_{s+1}$, satisfying the relations (12), have been established.

Put $x_{s+1}=\xi_{j}$. Existence and uniqueness of the point $x_{s+1}$, satisfying the relations (12), have been established. So, if $m_{s} \leq \varepsilon$, then we set $K=s$ and $x_{s+1}=b$ where in relation (13) holds. If $\varepsilon<m_{s}$, then there is a single point $\eta_{s+1}<b$ so that the inequality (12) is true. The induction transition is complete. This completes the proof. Obviously, the integer function $K(f, \varepsilon,|[a, b]|)$ has a monotonicity property: if $\varepsilon^{*}<\varepsilon^{\prime} \leq \varepsilon^{\prime \prime}<\varepsilon^{* *}$, then

$K\left(f, \varepsilon^{\prime},|[a, b]|\right) \geq K\left(f, \varepsilon^{\prime \prime},|[a, b]|\right)$.

Summing the relations (12), we obtain the inequality $\sum_{s=0}^{K-1} f\left(\left|\left[x_{s}, x_{s+1}\right]\right|\right) \leq \varepsilon K(f, \varepsilon,|[a, b]|)<$

$\sum_{s=0}^{K-1} f\left(\left|\left[x_{s}, x_{s+1}^{+}\right]\right|\right)$.

The set of segments $[c, d]$, contained in the segment $[a, b]$, we denote by $\mathcal{O}_{0}$. Consider any segment $[c, d]$ from $[a, b]$. Let $f_{0}:[c, d] \rightarrow R^{1}$ be mapping with the following properties:

$$
\begin{aligned}
f_{0}([c, d]) \geq 0 \quad \forall[c, d] \subset[a, b], \\
f_{0}([c, c])=0 \quad \forall c \in[a, b],
\end{aligned}
$$

$\mathcal{M} \subset \mathcal{M}^{\prime} \Rightarrow f(\mathcal{M}) \leq f\left(\mathcal{M}^{\prime}\right) \quad \forall \mathcal{M}, \mathcal{M}^{\prime} \subset \mathcal{O}_{0}(16)$ Moreover, suppose that for any $c \in[a, b]$ function $\phi_{s}(x)=f([c, x])$ of argument $x \in[c, b]$ is continuous:

$$
\begin{aligned}
& \phi_{s}(x-0)=\phi_{s}(x+0) \\
& \forall x \in(c, b) \forall c \in[a, b) .
\end{aligned}
$$

The mapping $f_{0}$ with properties (15)-(17) will be called a pseudo-measure on the segment $[a, b]$. 
Let the number $\varepsilon$ be in the interval $\left(\varepsilon^{*}, \varepsilon^{* *}\right)$,

$$
\varepsilon \in\left(\varepsilon_{*}, \varepsilon_{* *}\right)
$$

where

$$
\begin{aligned}
& \varepsilon_{*}=\max _{\xi \in\left[a, b^{-}\right]} f_{0}\left(\left[\xi, \xi^{+}\right]\right), \\
& \varepsilon_{* *}=\max _{\xi \in\left[a, b^{-}\right]} f_{0}([a, b]) .
\end{aligned}
$$

Consider the grid $Y \subset[a, b]$,

$$
\begin{aligned}
& Y=Y\left(f_{0}, \varepsilon,[a, b]\right) \text { : } \\
& \qquad=y_{0}<y_{1}<\ldots<y_{K_{0}}<y_{K_{0}+1}=b \\
& f_{0}\left(\left[y_{s}, y_{s+1}\right]\right)=\varepsilon, \forall s \in\left\{0,1, \ldots, K_{0}-1\right\}, \\
& \qquad f_{0}\left(\left[y_{K_{0}}, b\right]\right) \leq \varepsilon
\end{aligned}
$$$$
\text { such that }
$$

Definition 2. Grid (20) with properties (21)-(22) is called an adaptive grid (for $f_{0}$ on the segment $[a, b])$.

Theorem 2. If the relations (15)-(19) are satisfied, then there exist a natural number

$$
K_{0}=K_{0}\left(f_{0}, \varepsilon,|[a, b]|\right)
$$

and grid (20) with properties (21)-(22).

Proof is by induction on the number of nodes in the same way as this was done in the proof of Theorem 1. Detailed development proof is provided to the reader. For each grid segment $|[c, d]| \in \mathcal{O}$ we discuss a map

defined by the relation

$$
\tilde{f}:|[c, d]| \rightarrow R^{1}
$$

$$
\tilde{f}(|[c, d]|)=f_{0}(|[c, d]|) .
$$

It is easy to check that mapping $\tilde{f}$ satisfies properties (7)-(8) and, therefore, is a pseudomeasure on at the grid segment $|[a, b]|$. We say that the grid pseudo-measure $\tilde{f}$ is induced by the pseudo-measure $f_{0}$ defined on the segment $[a, b]$. Suppose we are considering a sequence of grids $|[a, b]|_{\lambda}$ of the form (6) with parameter $\lambda$ from the set $\Lambda \subset R_{+}^{1} \quad$ with zero condensation point. Suppose the maximum step $\tau_{\lambda}$ in the grid segment $\|\left.[a, b]\right|_{\lambda}$ tends to zero as $\lambda \rightarrow+0$. For each grid segment $\|\left.[a, b]\right|_{\lambda}$ consider grid pseudomeasure $\tilde{f}_{\lambda}$ induced pseudo-measure $f_{0}$ on the segment $[a, b]$. According to Lemma 1, we find number

$$
K_{\lambda}=K\left(\tilde{f}_{\lambda}, \varepsilon,|[a, b]|_{\lambda}\right)
$$

and grid

$$
X_{\lambda}=X\left(\tilde{f}_{\lambda}, \varepsilon, \|[a, b] \mid\right)=\left\{x_{s}(\lambda)\right\}_{s \in\left\{0,1, \ldots, K_{\lambda}\right\}}
$$

(see formula (11)) with properties (12) - (13).

By the definition of the pseudo-measure $\tilde{f}_{\lambda}$, and by formulas (23) - (25) the following statement follows easily.

Theorem 3. If for fixed $\varepsilon>0$ and for some $\lambda_{0}>0$ properties (12) - (13) are satisfied $\forall \lambda \in\left(0, \lambda_{0}\right)$ then the ratio $\lim _{\lambda \rightarrow+0} X_{\lambda}=Y$ is true. In other words,

$$
\begin{aligned}
& \lim _{\lambda \rightarrow+0} K\left(\widetilde{f}_{\lambda}, \varepsilon,|[a, b]|_{\lambda}\right)=K_{0}\left(f_{0}, \varepsilon,|[a, b]|\right) \\
& \lim _{\lambda \rightarrow+0} x_{s}(\lambda)=y_{s}, s \in\left\{0,1, \ldots, K_{\lambda}\right\} .
\end{aligned}
$$

\section{Computational Complexity of the Responsive Grid}

It is easy to see that the proof of Lemma 1 contains the algorithm for construction of the adaptive grid $X$. Consider the question of the complexity of this algorithm. Let $N(f)$ and $N_{\text {comp }}=N_{\text {comp }}(f)$ be a number of calculations pseudo-measures $f$ and the number of comparisons in the considered algorithm.

Theorem 4. Algorithm for construction of the adaptive grid $X$ has the following properties

$$
N(f)=N_{\text {comp }}(f)=K+M+2 \text { : }
$$

Proof. The adaptive grid can be written in the form $X=X\left(\tilde{f}_{\lambda}, \varepsilon, \|[a, b] \mid\right):$

$$
\begin{aligned}
a=x_{0}=\xi_{0}<x_{1} & =\xi_{p_{1}}<\ldots<x_{K}=\xi_{p_{K}}< \\
x_{K+1} & =\xi_{p_{K+1}}=b ;
\end{aligned}
$$

where $p_{K+1}=M+1$. The adaptive grid is shown in Fig.1. At the $s$-th step of this algorithm, we go from the node $x_{s}$ to the node $x_{s+1}$.

Suppose $x_{s}=\xi_{p_{s}}, x_{s+1}=\xi_{p_{s+1}}$.

It is not difficult to see that the mentioned transition requires:

1) calculate $p_{s+1}+p_{s}+1$ times the pseudo-measure $f \quad$ (on grid segments $\left|\left[\xi_{p_{s}}, \xi_{p_{s}+1}\right]\right|$, $\left|\left[\xi_{p_{s}} \xi_{p_{s}+2}\right]\right|, \ldots,\left|\left[\xi_{p_{s}}, \xi_{p_{s+1}+1}\right]\right|$,

2) a comparison of the result with $\varepsilon$ happens $p_{s+1}-$ $p_{s}+1$ times.

Since $s$ changes from 0 to $K$, then the total number $N(f)$ of calculations of the pseudo-measure $f$ is

$$
N(f)=\sum_{s=0}^{K}\left(p_{s+1}-p_{s}+1\right)=K+1+p_{K+1}-p_{0}
$$

According to the formulas (11) and (29), $p_{0}=0$, $p_{K+1}=M+1$, so as a result we get $N(f)=K+$ $M+2$. In the same way we find the number of $N_{\text {comp }}$ comparisons. So the formulas (28) are valid. This concludes the proof.

\section{Haar Approximation for Discrete Flow}

Suppose that a function $u(t)$ is given on the grid segment

$|[a, b]|: a=\xi_{0}<\xi_{1}<\ldots<\xi_{M}<\xi_{M+1}=b$

Let be

$$
a \leq y \leq t \leq b ; y, t \in|[a, b]| ; y=\xi_{i}, t=\xi_{j}
$$

From (30)-(31) we get 


$$
u(t)-u(y)=\sum_{s=i}^{j-1} \frac{u\left(\xi_{s+1}\right)-u\left(\xi_{s}\right)}{\xi_{s+1}-\xi_{s}}\left(\xi_{s+1}-\xi_{s}\right) .
$$

Lemma 1. For a discrete function $u \in|[a, b]|$ the inequality holds

$$
\begin{gathered}
|u(t)-u(y)| \leq \max _{\xi \in\left|\left[y, t^{-}\right]\right|}\left|D_{\Xi} u(\xi)\right|(t-y) \\
y \leq t, y, t \in|[a, b]|
\end{gathered}
$$

where

$$
D_{\Xi} u(\xi)=\frac{u\left(\xi^{+}\right)-u(\xi)}{\xi^{+}-\xi}
$$

Proof. Consider the relation (32), assuming $y=\xi_{i}$ and $t=\xi_{j}$. Taking into account the positiveness of the differences $\xi_{s+1}-\xi_{s}, t-y$ and notation (35), we see that the formulas (33) - (34) follow directly from the relation (32).

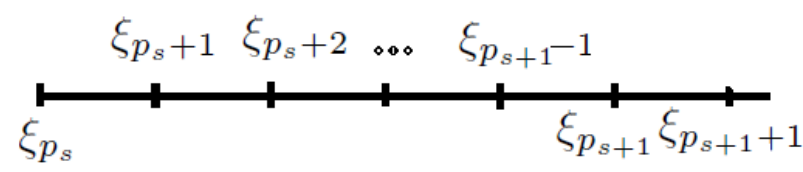

Fig.1. The adaptive grid.

\section{Grid Coarsening}

Consider a grid $\tilde{X}$, which is a subset of the grid $\Xi$

$\tilde{X}: a=\hat{x}_{0}<\cdots<\hat{x}_{K}<\hat{x}_{K+1}=b, \tilde{X} \subset \Xi$.

For the grid function $u(t)$ defined on $|[a, b]|$, we

construct a piecewise constant interpolation

$$
\tilde{u}(t)=u\left(\hat{x}_{j}\right) \forall t \in\left[\hat{x}_{j}, \hat{x}_{j+1}\right), j \in\{0,1, \ldots \widetilde{K}\} .
$$

Theorem 5. For a piecewise constant interpolation (37) for $t \in \mid\left[\hat{x}_{j}, \hat{x}_{j+1} \mid[\right.$ the inequalities hold

$$
\tilde{u}(t)-u(t)\left|\leq\left(\hat{x}_{j+1}-\hat{x}_{j}\right) \max _{\xi \in\left|\left[\hat{x}_{j}, \hat{x}_{j+1}{ }^{-}\right]\right|}\right| D_{\Xi} u(\xi) \mid \text {. }
$$

If $u \in C^{1}[a, b]$, then

$$
\widetilde{\mid u}(t)-u(t)\left|\leq \max _{\xi \in\left|\left[\hat{x}_{j}, \hat{x}_{j+1}\right]\right|}\right| u^{\prime}(\xi) \mid\left(\hat{x}_{j+1}-\hat{x}_{j}\right) \text {. }
$$

Proof. By formulas (33) - (37) for $y=\hat{x}_{j}$ and $t \in$ $\mid\left[\hat{x}_{j}, \hat{x}_{j+1} \mid[\right.$ we have

$$
\begin{aligned}
\widetilde{u}(t)-u(t) \mid & =\left|u(t)-u\left(\hat{x}_{j}\right)\right|=|u(t)-u(y)| \\
& \leq \max _{\xi \in\left|\left[y, t^{-}\right]\right|}\left|D_{\Xi} u(\xi)\right|(t-y) .
\end{aligned}
$$

To prove the inequality (38) it remains to return to the initial notation in the relation (40) and take advantage of the inequality $t<\hat{x}_{j+1}$. Inequality (39) follows from the Lagrange formula.

\section{Adaptive Grid Approximation}

Theorem 6. Let $\eta>0$ and the grid $\hat{X}$ is inconsistent with the grid $K(f, \eta,|[a, b]|)$, where $f$ is a map defined by the formula $f(|[c, d]|)=\max _{t \in|[c, d]|}\left|D_{\Xi} u(t)\right|(d-c)$

$\forall|[c, d]| \in \mathcal{O}$.
Then the following statements are true.

1.The number of nodes $K^{\prime}{ }_{u, \hat{X}}(\eta K(f, \eta,|[a, b]|)$ of this grid satisfies the relations

$\sum_{s=0}^{K-1} \max _{t \in\left|\left[x_{s}, x_{s+1}\right]\right|}\left|D_{\Xi} u(t)\right|\left(x_{s+1}-x_{s}\right) / \eta$.

2. The inequality is true

$$
\widetilde{u}(t)-u(t) \mid \leq \eta \forall t \in[a, b] .
$$

3. The algorithm for constructing an adaptive grid $X$ has the following properties

$$
N(f)=N_{\text {comp }}(f)=(K+M+2) .
$$

Proof. Note that the map (41), satisfies the properties (7) - (8). Thus, $f$ is a pseudo-measure. Formula (42) follows from the previously established formula (14), in which you need to take the pseudo-measure $f$ according to the relation (41) and set $\varepsilon=\eta$. The inequality (43) is obtained by the same substitutions $f$ and $\varepsilon$ from (12) and (38). Finally, the formula (44) is obtained from (28) with the mentioned choice pseudo-measures $f$. This concludes the proof.

Corollary. Formulas (28) and (41) show that in the conditions of Theorem 6 the number of arithmetical operations is directly proportional to the length of the initial flow.

\section{Illustrative Examples}

The purpose of the numerical experiment is to determine the effectiveness of the proposed adaptive algorithm for compression of the initial flow of numerical information. The initial flow $u\left(\xi_{i}\right)$ is generated by the function $u(t)$ using a uniform grid $\xi_{i}=i \hat{h}, \quad i=0,1, \ldots, M+1$, on the segment $[a, b], \hat{h}=(b-a) /(M+1)$. In this numerical experiment, we set $a=0, b=\pi, M+1=$ 1000. First, consider the standard compression $u\left(\xi_{2 j}\right)$ obtained by deleting nodes with odd numbers, $j=1,2, \ldots, N+1, N+1=500$. Let $\widetilde{U}(t)$ be a piecewise constant interpolation (interpolation spline of the zero order) constructed by points $\left(\xi_{2 j}, u\left(\xi_{2 j}\right)\right), j=0,1,2, \ldots, N+1$. The deviation of this interpolation from the original flow is denoted $\varepsilon$,

$$
\varepsilon=\max _{i=1,2,3, \ldots, N-1}\left|u\left(\xi_{2 i+1}\right)-\widetilde{U}\left(\xi_{2 i+1}\right)\right| \text {. }
$$

With the same uniform deviation $\varepsilon$ we obtain the compressed flow of length $K+1$ using the proposed adaptive algorithm of compression for the initial flow. Here we use $\varepsilon=\eta$ (see Theorem 5 and Theorem 6) with usual difference approximation of the first derivative. The ratio $(M+1) /(K+1)$ characterizes the degree of adaptive compression, the ratio 
$(N+1) /(K+1)$ characterizes the degree of efficiency of applying adaptive compression in comparison with standard double compression. In the digital experiment we discuss the generating functions $u(t)=\ln (t), 1 / t, \ln (t+1), 1 /(t+1)$ on the interval $[\hat{h}, \pi]$, and the function $u(t)=$ $\cot (t)$ on the interval $[0.05, \pi-0.05]$. In all cases we took $M+1=1000, N+1=500$. The results of the digital experiment are shown in the Table 1. The first column contains the number of discussed flow, the second column contains the function $u(t)$, which generates the flow, the third column contains the deviation $\varepsilon$ mentioned above, the fourth column gives the length $K+1$ of the compressed flow after applying the proposed adaptive algorithm of compression to the original flow. The fifth and sixth columns contain characteristic of the degree of adaptive compression, characteristic of the degree of efficiency for applying adaptive compression in comparison with standard double compression. The runtime of the proposed adaptive algorithm for the compression is placed in the seventh column.

Table 1. Computational results

\begin{tabular}{|l|c|c|c|c|c|c|}
\hline No & $u(t)$ & $\varepsilon$ & $K+1$ & $\frac{(M+1)}{K+1}$ & $\frac{(N+1}{K+1}$ & $\begin{array}{l}\text { Time } \\
(\text { sec. })\end{array}$ \\
\hline 1 & $t$ & 0.003 & 501 & 2 & 1 & 0.28 \\
\hline 2 & $\ln (t)$ & 0.405 & 19 & 52.7 & 26.4 & 0.26 \\
\hline 3 & $\ln (1+t)$ & 0.003 & 358 & 2.80 & 1.40 & 0.52 \\
\hline 4 & $1 / t$ & 53.0 & 7 & 143 & 71.6 & 0.31 \\
\hline 5 & $\frac{1}{t+1}$ & 0.003 & 202 & 4.96 & 2.48 & 0.23 \\
\hline 6 & $\cot (t)$ & 1.02 & 37 & 27.1 & 13.5 & 0.34 \\
\hline
\end{tabular}

Remarks to Table 1.

1. Instead of the first derivative, we used its approximation using the difference ratio.

2. For $\cot (\mathrm{t})$ the interval $(0.05, \pi-0.05)$ was implemented.

3.The program is written in the Maple-17 system (see [17]). The calculations were carried out on an HP 27-p251ur monoblock.

4. The calculation time for the definition of the number $\varepsilon$ in all cases was approximately 0.5 seconds.

5. For a flow of length $M+1=10^{6}$ for the generating function $u(t)=\cot (t)$ it turned out that $\varepsilon=0.026, K+1=1544$, compression ratios and efficiency equal to 648 and 324, respectively.

The results of this digital experiment lead to the following conclusions:

1. The proposed adaptive algorithm for compressing flows of numerical information in the case of rapidly changing flows, the compression ratio ranges from 16.4 to 11.1 .

2. In the case of slowly changing flows, the compression ratio is much lower: it fluctuates between 2.69 and 3.46 .

3 . The behaviour of the efficiency factor (relative to standard compression) similar to the behaviour of the compression ratio (this ratio is half the compression ratio).

4. The first row of the table contains the obvious expected result. The adaptive Haar compression for a linear function is obviously the same as the standard compression (this can serve as confirmation of the correct operation of the program).

5. In the case of an increase in the length of the original flow, the coefficients of compression and efficiency increase significantly: for a flow of length $M+1=10^{6}$ for the generating function $u(t)=$ $\cot (t)$ it turned out that $\varepsilon=0.026, K+1=$ 1544 , compression ratios and efficiency equal to 648 and 324, respectively (see paragraph 5 in Remarks to Table 1).

Tables 2, 3 show the computational results when $M=1000$ and 1000000 . Let us dwell in more detail on line 6 of Table 2 . The function $\frac{1}{\sqrt{t}}$ has a singularity at point 0 . Therefore, it was impossible to carry out calculations near the point 0 due to the growth of the function. The calculations were carried out starting from the second grid interval. The function $\frac{\sin (t)}{t}$ is of particular interest. The graphs of the functions $\frac{\sin (t)}{t}$ and $\frac{1}{\sqrt{t}}$ are shown in Fig. 2 and Fig. 3 respectively.

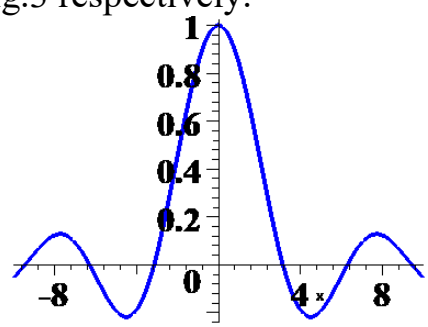

Fig.2. The plot of the function $\frac{\sin (t)}{t}$

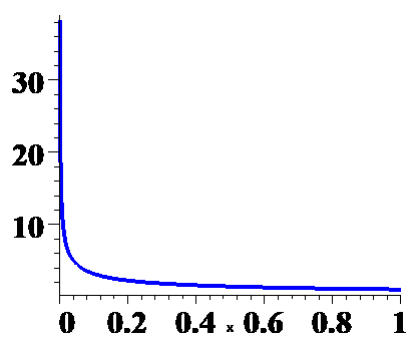

Fig.3. The plot of the function $\frac{1}{\sqrt{t}}$ 
Table 2. Computational results $M=1000$

\begin{tabular}{|l|c|c|c|c|c|c|}
\hline No & $u(t)$ & $\varepsilon$ & $K+1$ & $\frac{M+1}{K 1}$ & $\frac{N+1}{K+1}$ & $\begin{array}{l}\text { Time } \\
(\text { sec. })\end{array}$ \\
\hline 1 & $\sin (t)$ & 0.003 & 408 & 2.45 & 1.23 & 0.91 \\
\hline 2 & $\sin (10 \mathrm{t})$ & 0.031 & 420 & 2.38 & 1.19 & 0.37 \\
\hline 3 & $\sin (100 \mathrm{t})$ & 0.300 & 450 & 2.22 & 1.11 & 0.34 \\
\hline 4 & $\frac{\sin (t)}{t}$ & 0.001 & 440 & 2.28 & 1.14 & 0.34 \\
\hline 5 & $\frac{1}{t^{2}+1}$ & 0.002 & 315 & 3.2 & 1.6 & 0.30 \\
\hline 6 & $\frac{1}{\sqrt{t}}$ & 2.31 & 10 & 100 & 50 & 0.70 \\
\hline 7 & $\frac{1}{1+\sqrt{t}}$ & 0.01 & 38 & 25.7 & 12.8 & 0.36 \\
\hline
\end{tabular}

Table 3. Computational results $M=1000000$

\begin{tabular}{|l|c|l|l|l|l|l|}
\hline No & $u(t)$ & $\varepsilon$ & $K+1$ & $\frac{M+1}{K+1}$ & $\frac{N+1}{K+1}$ & $\begin{array}{l}\text { Time } \\
(\text { sec. })\end{array}$ \\
\hline 1 & $\sin (t)$ & $\begin{array}{l}6.4 \\
\cdot 10^{-5}\end{array}$ & $\begin{array}{l}3 \\
\cdot 10^{4}\end{array}$ & 32.7 & 16.3 & 29.3 \\
\hline 2 & $\sin (10 \mathrm{t})$ & $\begin{array}{l}6.0 \\
\cdot 10^{-4}\end{array}$ & $\begin{array}{l}3 \\
\cdot 10^{4}\end{array}$ & 32.6 & 16.3 & 33.0 \\
\hline 3 & $\sin (100 \mathrm{t})$ & $\begin{array}{l}6.0 \\
\cdot 10^{-3}\end{array}$ & $\begin{array}{l}3 \\
\cdot 10^{4}\end{array}$ & 32.3 & 16.2 & 33.3 \\
\hline 4 & $\frac{\sin (t)}{t}$ & $\begin{array}{l}2.0 \\
\cdot 10^{-4}\end{array}$ & $\begin{array}{l}46 \\
\cdot 10^{3}\end{array}$ & 21.4 & 10.7 & 31.0 \\
\hline 5 & $\frac{1}{t^{2}+1}$ & $\begin{array}{l}4.9 \\
\cdot 10^{-6}\end{array}$ & $\begin{array}{l}164 \\
\cdot 10^{3}\end{array}$ & 6.1 & 3.05 & 13.08 \\
\hline 6 & $\frac{1}{\sqrt{t}}$ & $\begin{array}{l}73.2 \\
12\end{array}$ & $\begin{array}{l}83 \\
\cdot 10^{3}\end{array}$ & $\begin{array}{l}42 \\
\cdot 10^{3}\end{array}$ & 25.0 \\
\hline 7 & $\frac{1}{1+\sqrt{t}}$ & $\begin{array}{l}0.6 \\
\cdot 10^{-3}\end{array}$ & 1132 & 883 & 441 & 25.9 \\
\hline
\end{tabular}

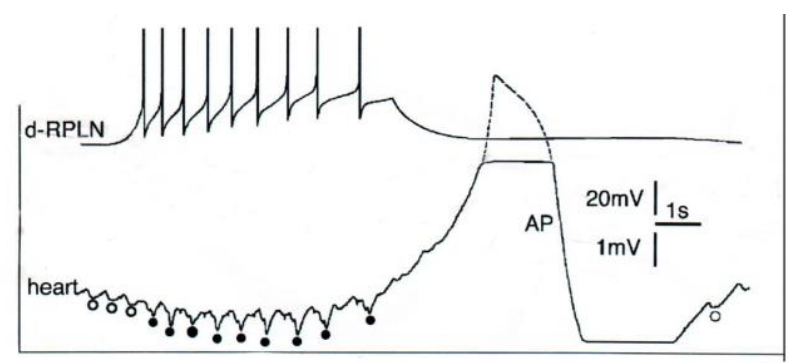

Fig. 4. The giant multimodal heart motoneurons of Achatina fulica

Let us also give an example of using the proposed approach in the case of processing information received from an analog device (see the lower graph with the name "heart" in Fig. 4). Digitization of the mentioned information resulted in the initial numerical stream of length $M=50$. Removing the odd nodes led to an error of $\varepsilon=6$ with the resulting stream of length $N=25$. The use of the proposed adaptive method led to a flow of length $K=4$ with the same precision $\varepsilon=6$. Thus, the efficiency of the resulting compression is $(\mathrm{M}+1) /(\mathrm{K}+1)=10$. At the same time, the burst observed on the graph was clearly presented. Fig. 4 (heart) shows the giant multimodal heart motoneurons of Achatina fulica (see [18], Fig.2b). The work uses modern methods of processing numerical information: spline-burst (wavelet) decomposition of information flows. The original stream was obtained by sampling the data supplied by the corresponding analog device. Spline-wavelet processing led to the appearance of the main and wavelet flows. The wavelet flow was considered as a noise component. Essential information was contained in the main thread. The length of the processed initial streams ranged from a several hundred to a thousand numbers, and the length of the corresponding main streams ranged from a several ten to a several hundred. The results obtained were in line with the expected. As an illustration, we processed the flow from an analog device that records the work of the heart of the snail under consideration (see the heart curve in Fig. 2). The quantity of numbers in the stream was $M=$ $50, \varepsilon=6$, the resulting main stream contained $K=$ 4 numbers, but the main spike in the curve was correctly reflected by the main stream.

Thus, let us once again emphasize the difference between the type of compression we have proposed and the traditional one. Let the uniform grid of nodes be constructed on the interval $[a, b]$. Let us assume that we have constructed a grid of $M$ equidistant nodes on the interval $[a, b]$. The traditional approach is to remove the odd nodes and leave only the even nodes. Thus, we have constructed a sparse mesh of nodes. The number of nodes in this new mesh is half that of the original. We construct an approximation separately on each grid interval on the sparse mesh of nodes and calculate the approximation error $\varepsilon_{i}$ on each of these intervals. Next, we find the maximum value among $\left|\varepsilon_{i}\right|$. Thus we find $\varepsilon=\max _{i}\left|\varepsilon_{i}\right|$. Our next task is to construct a sparser grid of nodes, keeping the approximation error $\varepsilon$. To achieve this goal, we will use such a property of the function as the rate of its change on different parts of the interval. If the function changes slowly over some interval, then on this interval we can remove more nodes, while maintaining the same order of error. 


\section{Conclusion}

The results obtained in this work allow the sender to efficiently compress the initial data flow and transmit the compression result to the recipient using (in the case of a slow change in the initial flow) insignificant communication channel resources. The computational complexity of the proposed adaptive algorithm is proportional to the length of the initial flow. That is very important in Physics and Engineering. In this paper the spaces of the Haar type on arbitrary irregular grids are considered. With a non-classic approach, the choice of a non-uniform grid determines the characteristics of the space to be designed with wavelet decomposition. Thus, it becomes a possible adaptive choice of design space depending on the incoming flow.

In contrast to the classical approach, this paper considers the possibility of the adaptive compressing of the initial flow. The adaptability is related to the rate of change of said flow and is reflected in the definition of the embedded grid. The apparatus of the research is based on the concept of pseudo-measure introduced in this paper. The benefits of this approach are confirmed by the numerical experiments presented here. The authors hope that the proposed approach will be useful for developing adaptive approximation algorithms in the multivariate case.

\section{References:}

[1] I. Singh, S. Kumar, Haar Wavelet Methods for Numerical Solutions of Harry Dym (HD), BBM Burger's and 2D Diffusion Equations, Bulletin of the Brazilian Mathematical Society, Vol.49, No 2, 2018, pp. 313-338. DOI: 10.1007/s00574-017-0055-7.

[2] N. Pervaiz, I. Aziz, Haar wavelet approximation for the solution of cubic nonlinear Schrodinger equations, Physica A: Statistical Mechanics and its Applications, Vol. 545, paper 123738 , 2020, DOI: 10.1016/j.physa.2019.123738.

[3] A Raza, A. Khan, Approximate solution of higher order two point boundary value problems using uniform Haar wavelet collocation method, Springer Proceedings in Mathematics and Statistics, 272, 2019, pp. 209220. DOI: 10.1007/978-981-13-9608-3_14.

[4] R. Amin, S. Nazir, I. García-Magariño, A collocation method for numerical solution of nonlinear delay integro-differential equations for wireless sensor network and internet of things Sensors (Switzerland),Vol 20,7, paper 1962, 2020.

[5] E.H.S. Diop, A.-O. Boudraa, V.B.S Prasath, Optimal Nonlinear Signal Approximations Based on Piecewise Constant Functions, Circuits, Systems, and Signal Processing, Vol. 39, No 5, 2020, pp. 2673-2694. DOI: 10.1007/s00034-019-01285.

[6] A.Haar Zur, Theorie der orthogonalen Funktionensysteme, Mathematische Annalen, 69, 1910, pp. 331-371.

https://link.springer.com/article/10.1007/BF014 56326

[7] S.Mallat A Wavelet Tour of Signal Processing, Academic Press, 1999. DOI: 10.2118/96553MS.

[8] Francois Dubeau, Said Elmejdani, Riadh Ksantini, Non-uniform Haar wavelets, Applied Mathematics and Computation, Vol.159, 2004, pp. 675-691,

https://www.sciencedirect.com/science/article /abs/pii/S009630030301155X

[9] Yu. K.Demyanovich, A.Yu.Ponomareva, Adaptive Spline-Wavelet Processing of a Discrete Flow, J. Math. Sci., New York, Vol. 210, No 4, 2015, pp.371-390. DOI: 10.1007/s10958-015-2571-6.

[10] H, Saxena, A. Singh, J.N. Rai, Adaptive spline-based PLL for synchronisation and power quality improvement in distribution system, IET Generation, Transmission and Distribution, Vol. 14, No 7, 2020, pp. 13111319.

[11] D.Cornel, A.Buttinger-Kreuzhuber, A.Konev, Z.Horvath, M.Wimmer, R. Heidrich, J.Waser, Interactive visualization of flood and heavy rain simulations, Computer Graphics Forum, Vol.38, No 3, 2019, pp. 25-29.

[12] Z.Yang, H.Liu, T.Bi, Z.Li, Q.Yang, An adaptive PMU missing data recovery method, International Journal of Electrical Power and Energy Systems, Vol.116, paper 105577, 2020.

[13] I.G.Burova, E.F.Muzafarova, D.E.Zhilin, About adaptive grids construction, WSEAS Transactions on Mathematics, Vol.17, 2018, pp. 340-351.

[14] I.G.Burova,I.I.Narbutovskikh, E.F.Muzafarova, Image Processing and the Spline Approximation of the Third and Fifth Order, International Journal of Circuits, Systems and Signal Processing, Vol. 13, 2019, pp. 550- 557.

[15] I.G.Burova,E.F.Muzafarova,I.I.Narbutovskikh, Local splines of the Second and Third Order, Complex-valued Splines and Image Processing, 
pp. 419-429, International Journal of Circuits, Systems and Signal Processing, Vol. 13, 2019.

[16] Yu. K. Dem'yanovich, General Flows and their Adaptive Decompositions, WSEAS Transactions on Mathematics, Volume 17, 2018, pp. 28-34.

[17] Maple 2017.0, Product Build IDs, Maple Build ID 1231047, Licensed to: Prof. Yuri Demyanovich, Serial Number:

M4SUJR24AKMC7YDY, Permanent Licence.

[18] Vladimir Zhuravlev, Vladislav Bugaj, Sodikdjon Kodirov, Tatiana Safonova, Alexandr Staruschenko, Giant multimodal heart motoneurons of Achatina fulica: a new cardioregulatory input in pulmonates,

Comparative Biochemistry and Physiology Part A, Vol. 130, 2001, pp.183-196.

\section{Creative Commons Attribution License 4.0 (Attribution 4.0 International, CC BY 4.0)}

This article is published under the terms of the Creative Commons Attribution License 4.0

https://creativecommons.org/licenses/by/4.0/deed.en US 